\title{
Platelet/Lymphocyte ratio independently predicts the outcome of severe aplastic anemia patients treated with antithymocyte globulin
}

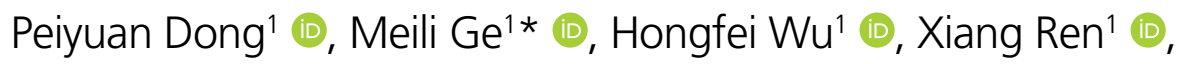

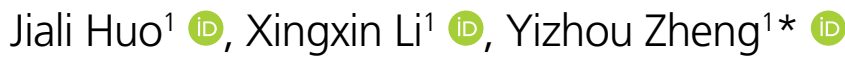

\begin{abstract}
SUMMARY
OBJECTIVE: The aim of this study was to determine the clinical role of platelet/lymphocyte ratio and neutrophi/lymphocyte ratio in severe aplastic anemia patients treated with antithymocyte globulin.

METHODS: The outcomes of consecutive severe aplastic anemia patients treated with rabbit or swine antithymocyte globulin plus cyclosporine ( $n=159$, from January 2012 to December 2018) were analyzed retrospectively.

RESULTS: In a total of 159 patients, the actuarial 5-year survival rate was $85.6 \%$. Low platelet/lymphocyte ratio (PLR $\leq 55)$ was significantly associated with less complications at 1 month and 24 months after the antithymocyte globulin treatment ( $p=0.048$ and 0.028 , respectively). The univariate and multivariate analyses revealed that low platelet/lymphocyte ratio was an independent predictor of overall survival $(p=0.03$ and 0.04 , respectively). Patients with low neutrophil/lymphocyte ratio (NLR $\leq 0.18)$ had shorter survival time, but there was no significant difference $(p=0.056)$. PLR was positively correlated with neutrophil/ymphocyte ratio $(r=0.38, p<0.0001)$ and age $(r=0.17$, $p=0.0379)$, while it was negatively correlated with $\lg G$ level $(r=-0.18, p=0.0309)$. The ratio of $C D 4 / C D 8$ was significantly higher in low platelet/lymphocyte ratio group $(\mathrm{p}=0.005)$.

CONCLUSION: The platelet/lymphocyte ratio reflects the immune abnormality of SAA. Notably, low platelet/lymphocyte ratio is an independently positive prognostic factor for severe aplastic anemia patients treated with antithymocyte globulin.

KEYWORDS: Anemia, aplastic. Antithymocyte globulin. Neutrophils. Platelet. Lymphocyte.
\end{abstract}

\section{INTRODUCTION}

Acquired aplastic anemia (AA) is primarily ascribed to activated $T$ lymphocytes, which induce destruction to hematopoietic stem cells, while the antigenic exposure leading to the expansion of dysregulated $\mathrm{CD}^{+}{ }^{+} \mathrm{T}$-cell populations ${ }^{1}$. For severe AA (SAA) patients, who are transfusion-dependent and in the absence of a human leukocyte antigen (HLA)-matched sibling, antithymocyte globulin (ATG) is recommended ${ }^{2}$. Young and less severe disease had a much higher probability of 10 -year survival, but none of them significantly predicted response to ATG at 6 months ${ }^{3}$. Although patients with baseline absolute reticulocyte count (ARC) $\geq 25 \times 10^{9} / \mathrm{L}$ and absolute lymphocyte count (ALC) $\geq 1 \times 10^{9} / \mathrm{L}$ had a better response to ATG at 6 months, they did not significantly predict the overall survival

'Peking Union Medical College, Chinese Academy of Medical Sciences, Institute of Hematology \& Blood Diseases Hospital, State Key Laboratory of Experimental Hematology - Tianjin, China.

*Corresponding authors: zheng_yizhou@hotmail.com, gemeili503@126.com

Conflicts of interest: the authors declare there are no conflicts of interest. Funding: This work was supported by grants from the National Natural Science Foundation of China (Grant No. 81700120 and 81770119).

Received on October 22, 2020. Accepted on October 22, 2020. 
(OS) in children ${ }^{4}$. Thus, how to predict the response and outcome of AA patients treated with ATG remained to be further investigated.

Further evidence indicated that autoimmune and inflammatory processes induced the apoptosis of $\mathrm{CD} 34^{+}$progenitor cells, influencing the disease course as well as response rate to ATG in AA patients. ${ }^{5}$. Platelet/lymphocyte ratio (PLR) and neutrophil/lymphocyte ratio (NLR) are novel markers of systemic inflammation, which have shown to be effective predictors of prognosis and therapy response in various immune diseases such as systematic lupus erythematosus and dermatomyositis $^{6,7}$. In hematological disease, PLR and NLR were also relevant to poor prognosis in peripheral T-cell lymphoma and diffuse large B-cell lymphoma ${ }^{8,9}$. In this study, it was suggested that baseline PLR and NLR were associated with lymphocyte subsets. The ratio of CD4/CD8 was significantly higher in low PLR group. However, to the best of our knowledge, no study till date has investigated the association between PLR as well as NLR and the prognosis of SAA patients treated with ATG. In this study, we retrospectively analyzed 159 SAA patients treated with ATG and intended to assess the prognostic roles of PLR and NLR in treatment-naïve SAA cases.

\section{METHODS}

\section{Study design}

This study is to determine the clinical role of PLR and NLR in SAA patients treated with ATG.

\section{Participants}

The eligibility criteria were as follows:

1. newly diagnosed SAA patients older than 2 years of age;

2. patients treated with first-line ATG from January 2012 to December 2018 at Blood Diseases Hospital, Chinese Academy of Medical Sciences;

3. patients or legal guardians were informed of the study which was approved by the Institutional Committee for Medical Care and Safety, and therefore performed in accordance with Declaration of Helsinki.

All the data were collected through the follow-up in the outpatient department or phone call until December 2019.

\section{Variables}

The definition and disease severity of AA patients included were defined according to the Camitta criteria ${ }^{10}$.

Two types of ATG regimens were included in this analysis:
1. rabbit ATG (rATG) (Thymoglobulin ${ }^{\circledR}$, Genzyme, Sanofi Company, Cambridge, MA, USA) was administered at a dose of $1.97 \mathrm{mg} / \mathrm{kg}$ per day for 9 days;

2. swine ATG (sATG) (Wuhan Institute of Biological Products Co., Ltd., Wuhan, China) at a dose of 20-30 mg/kg per day for 5 days, as previously described ${ }^{11}$. Cyclosporine was administered at a dose of $5 \mathrm{mg} / \mathrm{kg}$ per day $(3 \mathrm{mg} / \mathrm{kg}$ per day for children under 18 years of age), which was adjusted according to the serum creatinine levels.

\section{Data sources/measurement}

Both PLR and NLR were calculated by dividing the platelet count and absolute neutrophil count by the lymphocyte count, respectively. The data were collected at the time of diagnosis without transfusion of platelet and recombinant human granulocyte colony-stimulating factor (rhuG-CSF) treatment within 1 week.

\section{Statistical methods}

The optimal cut-off values for classifying NLR and PLR as low or high for the subsequent analysis were 0.18 and 55, respectively, which was determined using the receiver operating characteristic curve analysis for OS. The Mann-Whitney $U$ test and $\chi^{2}$ test were used to compare continuous and categorical variables, respectively. OS correlations were assessed using Kaplan-Meier curve with log-rank statistics. Furthermore, the univariate and multivariate Cox regression analyses were performed to calculate their respective hazard ratios (HRs) and 95\% confidence intervals (CIs). The statistical analyses were performed using SPSS software (version 22; IBM Corp., Armonk, NY, USA). $\mathrm{p}<0.05$ was considered as statistically significant.

\section{RESULTS}

\section{Participants}

Between 2012 and 2018, 159 SAA patients treated with ATG were included for the evaluation in this study.

\section{Descriptive data}

The characteristics of all the study participants were included in Table 1.

\section{Outcome data}

The number of patients who died within 3 months from the ATG treatment (early mortality) was 6 (3.8\%), and 14 (9.6\%) patients died beyond day +90 (late mortality). The causes of early mortality were infections $(n=5)$ and hemorrhage $(n=1)$. 
Table 1. Clinical characteristics of 159 SAA patients.

\begin{tabular}{|c|c|}
\hline Factors & Values \\
\hline Patients, $n$ & 159 \\
\hline Median age (years, range) & $22(3-57)$ \\
\hline \multicolumn{2}{|l|}{ Age groups } \\
\hline$<20$ years, $\mathrm{n}(\%)$ & $70(44)$ \\
\hline 20-40 years, $n(\%)$ & $68(42.8)$ \\
\hline$>40$ years, $n(\%)$ & $21(13.2)$ \\
\hline \multicolumn{2}{|l|}{ Gender, n (\%) } \\
\hline Male & $87(54.7)$ \\
\hline Female & $72(45.3)$ \\
\hline \multicolumn{2}{|l|}{ Etiology, n (\%) } \\
\hline Idiopathic & $156(98.1)$ \\
\hline Post-hepatitis & $3(1.9)$ \\
\hline \multicolumn{2}{|l|}{ Severity, n (\%) } \\
\hline SAA & $80(50.3)$ \\
\hline VSAA & $79(49.7)$ \\
\hline $\begin{array}{l}\text { Median follow-up after ATG } \\
\text { (months), mean (range) }\end{array}$ & $41(1-92)$ \\
\hline \multicolumn{2}{|l|}{ Peripheral blood counts } \\
\hline $\mathrm{Hb}$ (g/L), (median, range) & $30(76-131)$ \\
\hline PLT (×10/L), (median, range) & $21(1-115)$ \\
\hline ANC (×109/L), (median, range) & $0.43(0.01-4.36)$ \\
\hline ARC (×10\% $/ L)$, (median, range) & $9.3(0.54-128.9)$ \\
\hline ALC (×109/L), (median, range) & $1.35(0.13-5.29)$ \\
\hline \multicolumn{2}{|l|}{ NLR } \\
\hline$\leq 0.18, \mathrm{n}(\%)$ & $49(30.8)$ \\
\hline$>0.18, n(\%)$ & $110(69.2)$ \\
\hline \multicolumn{2}{|l|}{ PLR } \\
\hline$\leq 55, \mathrm{n}(\%)$ & $140(88.1)$ \\
\hline$>55, \mathrm{n}(\%)$ & $19(11.9)$ \\
\hline \multicolumn{2}{|l|}{ PNH clone, n (\%) } \\
\hline Positive & $25(15.7)$ \\
\hline Negative & $134(84.3)$ \\
\hline \multicolumn{2}{|l|}{ ATG agent, n (\%) } \\
\hline rATG & $98(61.6)$ \\
\hline sATG & $61(38.4)$ \\
\hline $\begin{array}{l}\text { Interval from diagnosis to ATG } \\
\text { (days) (median, range) }\end{array}$ & $32(10-134)$ \\
\hline
\end{tabular}

SAA: severe aplastic anemia; VSAA: very severe aplastic anemia; PLT: platelet count; ANC: absolute neutrophil count; ARC: absolute reticulocyte count; ALC: absolute lymphocyte count; NLR, neutrophil/ lymphocyte ratio; PLR: platelet/lymphocyte ratio; PNH: paroxysmal nocturnal hemoglobinuria; rATG: rabbit antithymocyte globulin; sATG: swine antithymocyte globulin.
In this study, 38\% (60/158) of patients showed complete and partial responses (CR and PR) within 3 months. At 6 months, the overall response rate (CR and PR) was $57.2 \%$ (83/145). Until the end of follow-up, the best response rate was 71.7\% (114/144).

The strongest predictor of response was the severity of disease; the response rate was $25.3 \%$ at 3 months and $41.4 \%$ at 6 months in very severe AA (VSAA) patients compared with $50.6 \%$ at 3 months and $72 \%$ at 6 months in SAA patients (HR=0.3; $\mathrm{p}=0.001$ and $\mathrm{HR}=0.3 ; \mathrm{p}<0.001$, respectively). The second prognostic factor was the diagnosis-to-treatment interval, $0-32$ or $>32$ days from diagnosis; the response rates at 3 months were $46.9 \%$ and $28.6 \%(\mathrm{HR}=2.2 ; \mathrm{p}=0.018)$.

The most common complications after ATG treatment within 1 month were infection $(25.8 \%)$ and hemorrhage (5.7\%). Low PLR $(\leq 55)$ predicted less complications after ATG treatment within 1 month and at 24 months: 30 versus $52.6 \%(\mathrm{p}=0.048)$ and 4.2 versus $33.3 \%(\mathrm{p}=0.028)$, respectively.

\section{Main results}

The platelet/lymphocyte ratio was positively correlated with NLR $(\mathrm{r}=0.38,95 \% \mathrm{CI} 0.23-0.50, \mathrm{p}<0.0001)$ and age $(\mathrm{r}=0.17,95 \% \mathrm{CI}$ $0.01-0.31, \mathrm{p}=0.0379)$. However, PLR was negatively correlated with IgG level ( $\mathrm{r}=-0.18,95 \% \mathrm{CI}-0.33--0.02, \mathrm{p}=0.0309)$.

There was a significant difference in lymphocyte subsets between low PLR group and high PLR (PLR $>55)$ group (Figure 1). The ratio of lymphocyte/nucleated cell, $\mathrm{CD} 3^{+} \mathrm{CD}^{+}$ cell/lymphocyte, $\mathrm{CD} 19^{+}$cell/lymphocyte, and $\mathrm{CD} 4 / \mathrm{CD} 8$ was found to be significantly higher in low PLR group (78.5 versus $57.8 \%, \mathrm{p}<0.0001 ; 42.2$ versus $31.3 \%, \mathrm{p}=0.001 ; 14.3$ versus $8.5 \%, \mathrm{p}=0.028 ; 1.7$ versus $1.1, \mathrm{p}=0.005$, respectively), while the ratio of $\mathrm{CD} 3{ }^{+} \mathrm{CD} 8{ }^{+}$cell/lymphocyte and $\mathrm{CD} 3{ }^{-} \mathrm{CD} 56^{+}$ cell/lymphocyte tended to be higher in high PLR group ( 34.2 versus $28.7 \%, p=0.023 ; 15.0$ versus $7.3 \%, p<0.0001$, respectively). Only the ratios of lymphocyte/nucleated cell and $\mathrm{CD} 3{ }^{+} \mathrm{CD} 4{ }^{+}$cell/lymphocyte were significantly higher in $\mathrm{NLR} \leq 0.18$ group $(88.6$ versus $70.1 \%, \mathrm{p}<0.0001 ; 45.3$ versus $38.6 \%, \mathrm{p}=0.005$, respectively). There was no significant difference between $N L R \leq 0.18$ group and NLR $>0.18$ group in other lymphocyte subsets.

With a median follow-up of 41 months (range: 0-92 months), the OS rate at 5 years was $85.6 \pm 3.3 \%$ (Figure 2A). Posttreatment status at 3 months strongly predicted the long-term survival; the 5 -year OS rates of patients with response and no response were $96.5 \pm 2.4 \%$ and $75.3 \pm 5.4 \%(\mathrm{p}=0.002)$; low PLR predicted a higher 5 -year survival $(87.0 \pm 3.2 \%$ versus $71.8 \% \pm 10.7 \%, \mathrm{p}=0.03$ ) (Figure $2 \mathrm{C}$ ); patients with ALC $>1 \times 10^{9} / \mathrm{L}$ at diagnosis had significantly higher 5 -year OS (90.1 $\pm 3.2 \%)$, as compared with patients with ALC $\leq 1 \times 10^{9} / \mathrm{L}(74.4 \pm 6.8 \%, \mathrm{p}=0.018)$; the severity of disease was also predictive, with a 5 -year survival of $90.7 \pm 3.7 \%$ and $79.1 \pm 5 \%$ 


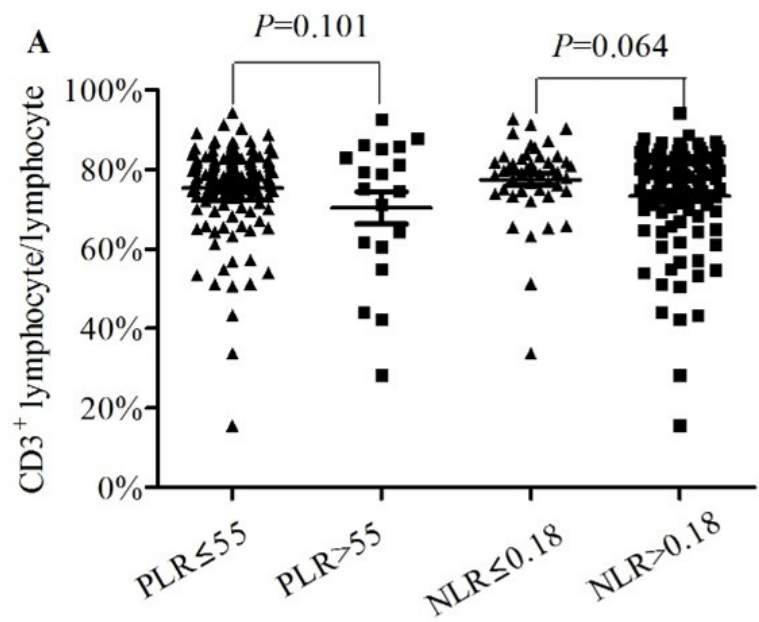

B

C
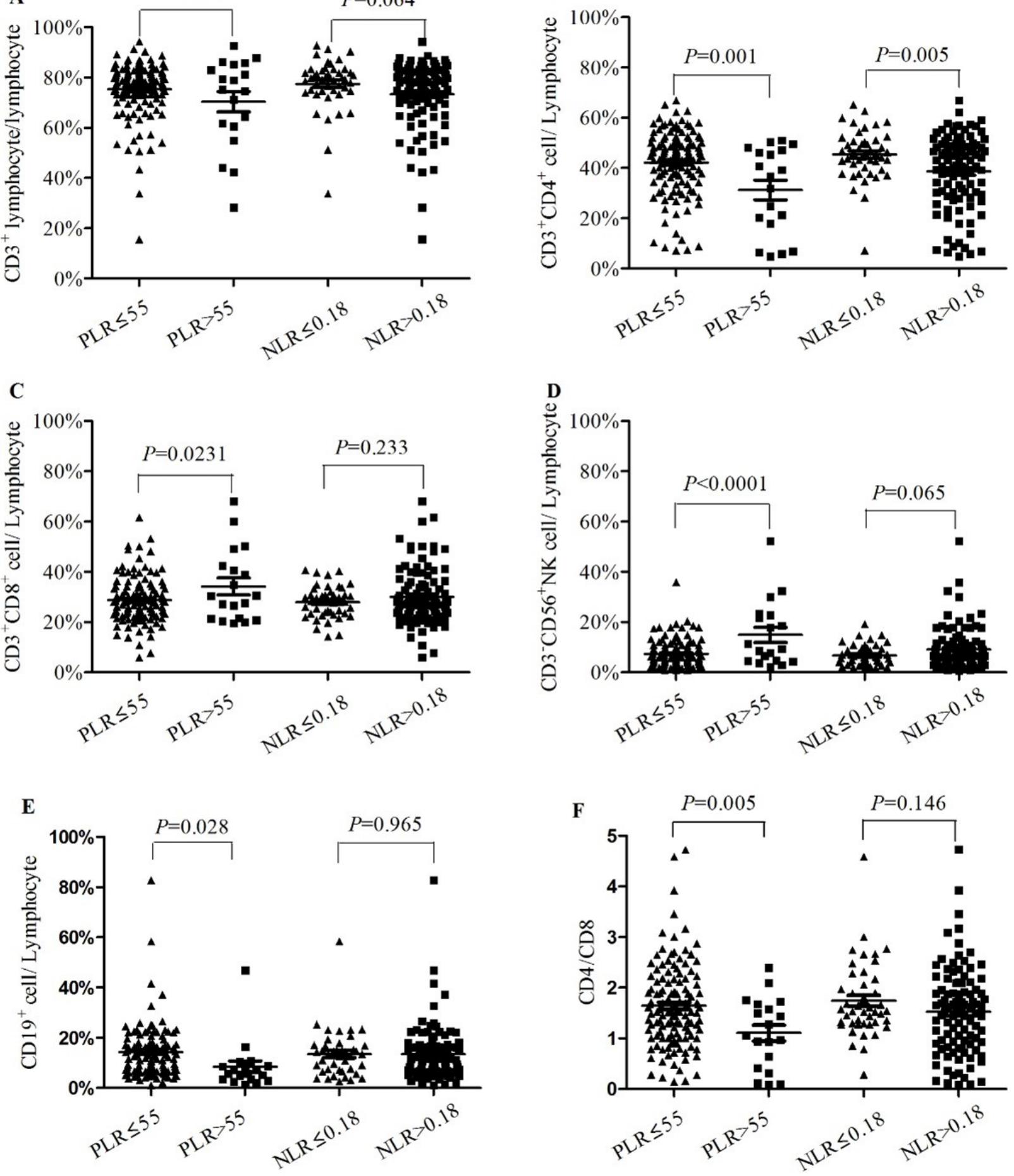

Figure 1. Baseline PLR and NLR correlated to lymphocyte subsets. Neither PLR nor NLR correlated with the percentage of $\mathrm{CD}^{+}$lymphocyte/lymphocyte (A). Patients with PLR $\leq 55$ had higher $C D 3^{+}$CD4+ lymphocyte/lymphocyte (B), lower CD3+CD8+ lymphocyte/lymphocyte (C), lower CD3+CD56+ NK cell/lymphocyte (D), higher CD19+ cell/lymphocyte (E), and higher CD4/ CD8 (F). PLR, platelet/lymphocyte ratio; NLR, neutrophil/lymphocyte ratio. 


\section{$\mathbf{A}$}

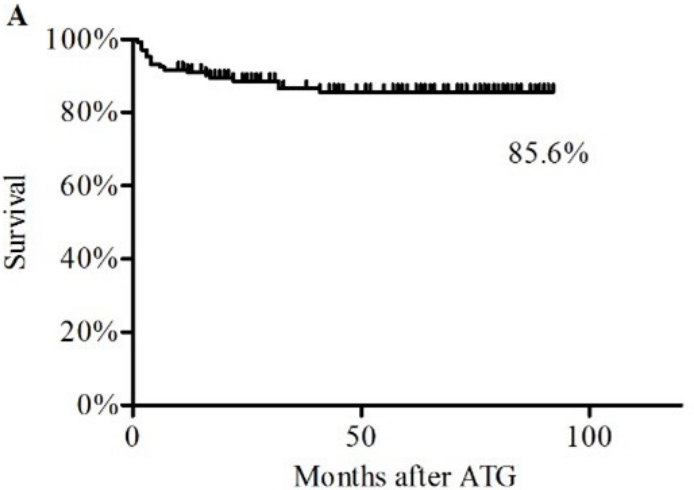

C

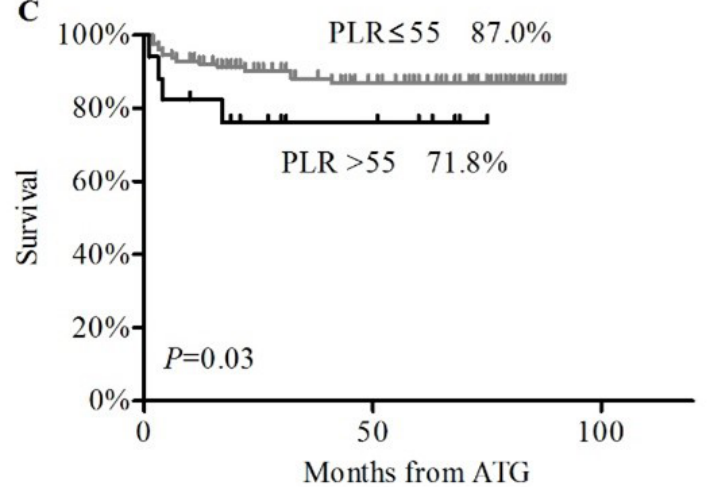

$\mathbf{E}$

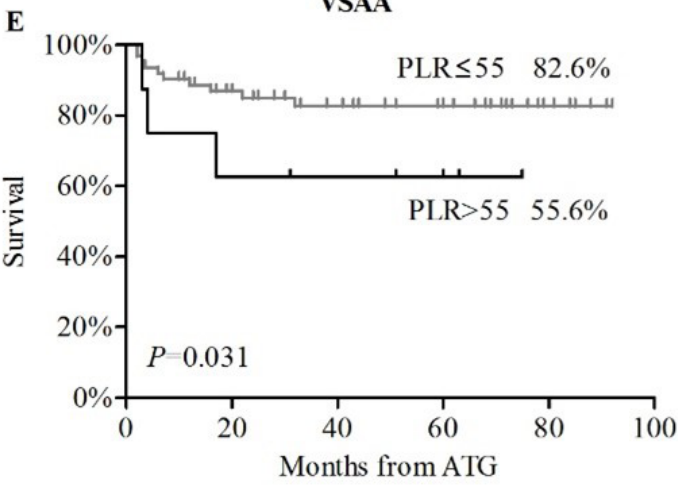

B

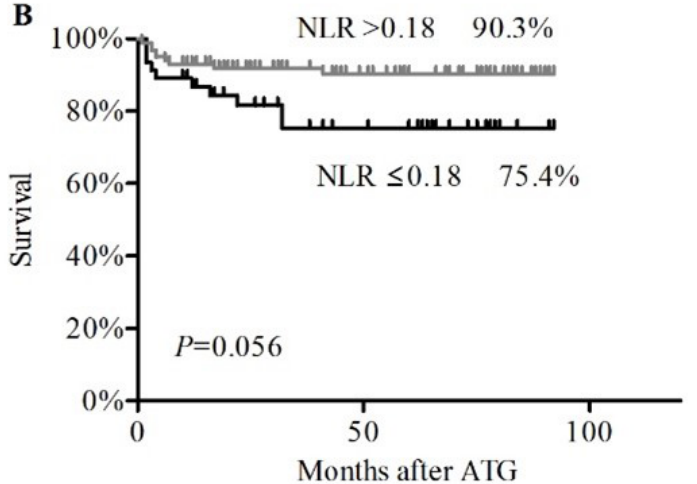

D

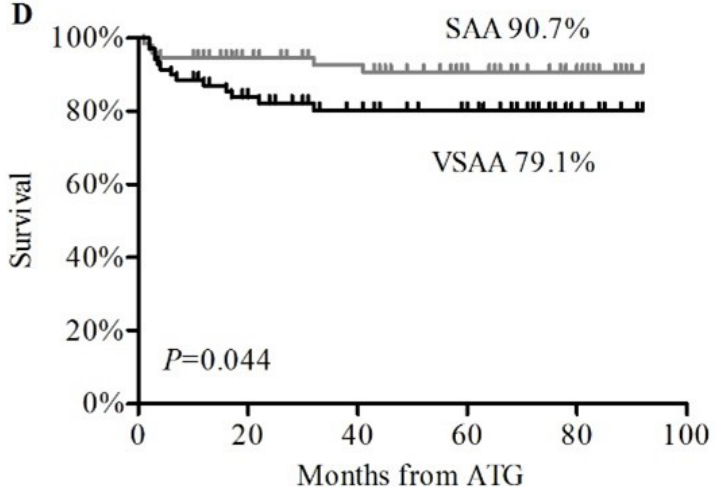

F

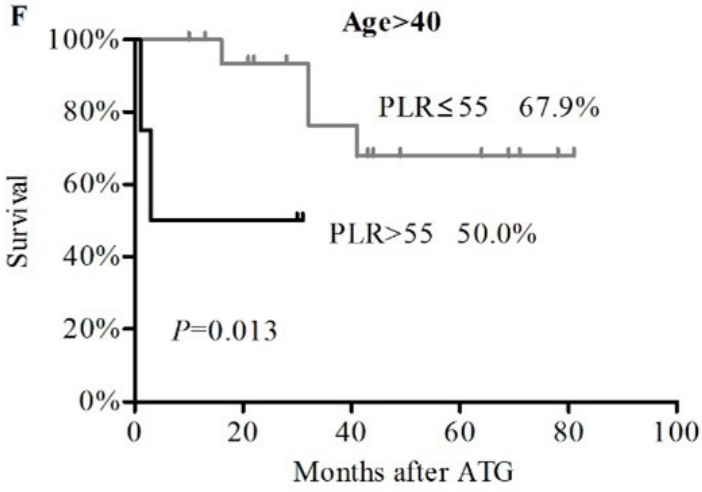

Figure 2. Kaplan-Meier curves of overall survival. (A) Five-year overall survival of 159 patients with AA treated with ATG. (B-D) Survival of patients stratified according to NLR, PLR, and severity of disease: a significant positive effect was seen for patients with PLR $\leq 55$ and SAA patients. (E and F) Comparison of the impact of PLR on overall survival according to the severity of disease and age separately. AA, aplastic anemia; ATG, antithymocyte globulin; NLR, neutrophi//ymphocyte ratio; PLR, platelet/lymphocyte ratio; SAA, severe aplastic anemia; VSAA, very severe AA.

for SAA and VSAA patients ( $\mathrm{p}=0.044$ ) (Figure 2D), respectively. However, age, NLR, ARC, and paroxysmal nocturnal hemoglobinuria $(\mathrm{PNH})$ clone did not significantly affect the OS.

As the severity of disease was a significant predictor of prognosis, we classified the patients into two groups: SAA and VSAA. Low PLR (PLR 555$)$ was still a positive predictor in VSAA group (Figure 2E). Although PLR was positively correlated with age as described earlier, low PLR still predicted the better prognosis in patient group aged $>40$ (Figure $2 \mathrm{~F}$ ).

Since PLR was significantly correlated with age, both ALC and NLR factors were excluded in the multivariate analysis. Factors such as gender, PLR, ARC, PNH, and the severity of disease were included. As expected, PLR was the independent predictive factor for patients treated with ATG [HR=0.34, (95\%CI 0.12-0.95), $\mathrm{p}=0.04]$. 


\section{DISCUSSION}

Antithymocyte globulin (ATG) is often recommended as a first-line therapy for AA patients who lack a matched sibling donor, especially for SAA and VSAA patients. In this study, the 3-month (37.7\%), 6-month (52.2\%), and best response rates $(71.7 \%)$ were higher than the previous reports ${ }^{12,13}$, respectively, due to our optimized ATG therapy ${ }^{11}$. The 5-year survival and early mortality for AA patients with ATG were 85.6 and 3.8\%, respectively, and in our center, the results were compared with those reported in the earlier foreign studies ${ }^{3,14}$.

In this cohort, we determined that the patients with diagnosis-to-treatment interval $<32$ days had better response to ATG at 3 months than those with longer ones ( 46.9 versus $28.6 \%$, $\mathrm{p}=0.018)$. Response to ATG in VSAA patients at 3 months was worse than that of SAA patients ( 25.3 versus $50.6 \%$, $\mathrm{p}<0.001$ ). But there were no significant difference in other factors such as age, ARC, ALC, NLR, and PNH clone. These results were consistent with the long-term follow-up consequences of a multicenter study in Europe and Asia ${ }^{3}$.

Various studies had investigated the risk factors that predicted the OS: age, the diagnosis-to-treatment interval, severity of AA, ALC, ARC, PNH clone, and telomere length ${ }^{3,12,14-16}$. In consistent with the previous studies ${ }^{3,4}$, we determined that ALC $>1 \times 10^{9} / \mathrm{L}$ and SAA were correlated with longer survival. Although an evidence suggested that a minor population of PNH-type cells and ARC was the positive predictors of $\mathrm{ATG}^{16,17}$, other studies showed that there was no difference in outcome related to these factors ${ }^{4,18}$. Thus, until now, there are no sufficient practical and reliable factors to predict the outcomes of ATG therapy.

At present, this is the first study to investigate the relationship between PLR as well as NLR and the outcomes of AA patients treated with ATG. More importantly, we found that low baseline PLR level (PLR $\leq 55)$ in SAA patients was an independent positive prognostic factor $(H R=0.34, p=0.04)$. Since PLR and NLR were the novel markers of systemic inflammation, we determined that baseline PLR and NLR were associated with lymphocyte subsets in our data. Besides, PLR was positively correlated with NLR and age, however it was negatively associated with IgG level.

Lymphocytes play a central role in the pathogenesis of AA. Activated T lymphocytes mediate autoimmunity through producing pro-inflammatory cytokines and thereafter lead to bone marrow destruction ${ }^{1}$. ATG is a purified antibody, which can ablate abnormal $\mathrm{T}$ lymphocytes as well as reverse the "immune-mediated" pathogenic mechanisms in $\mathrm{AA}^{19}$. As expected, we demonstrated that the patients with low PLR have better prognosis. It is worth to mention that these patients had higher ratio of CD4/CD8. Indeed, $\mathrm{CD}^{+}{ }^{+}$cells played an important role in the pathogenesis of AA, especially Th 1 cells. A polarization of $\mathrm{CD}^{+}$cells toward a type-1 response leads to the activation of cytotoxic $\mathrm{CD} 8^{+}$cells and finally to hematopoietic stem cell destruction ${ }^{20}$. Thus, we hypothesized that PLR as well as NLR predicted the abnormal quality and function of lymphocyte subsets, and low PLR was more likely correlated with severe immunity of this disorder. Therefore, it is plausible and necessary to include PLR and NLR into factors predicting the outcomes of ATG.

There were some limitations in this study. First, this study was based on the retrospective data collection and completed at a single center. Second, the patients in this study were included with rATG and sATG. However, there was no significant difference between them as studied previously ${ }^{21}$.

Despite the above-mentioned limitations, this study had some clinical implications. First of all, this is the first study that evaluates the role of PLR and NLR in the treatment of SAA. Second, we found that PLR could predict initial response to ATG treatment of SAA patients. Low PLR was a positively significant predictor of better response, fewer complications, and longer survival after ATG. Furthermore, PLR and NLR were closely related to lymphocyte subsets, which reflected immune abnormality in SAA patients. This finding might guide clinicians to judge the treatment response of SAA patients to ATG earlier and determine better therapy scheme, which has the meaningful and useful implications for clinical practice. Finally, PLR can be acquired at low cost in clinical practice, so it has potential to be a simple, convenient predictive, and stratification factor to assist with clinical decision-making for SAA patients.

\section{CONCLUSION}

The platelet/lymphocyte ratio reflected immune abnormality in SAA patients, and a low PLR was an independently significant predictor of SAA patients treated with ATG.

\section{ACKNOWLEDGMENT}

The authors would like to thank all the doctors and nurses in the Therapeutic Centre of Anemic Diseases and the researcher team of the Clinical Laboratory Centre for their professional assistance.

\section{ETHICAL APPROVAL}

The retrospective protocol of this study was approved by the ethics review board of Institute of Hematology and Blood Diseases Hospital, Chinese Academy of Medical Science and Peking Union Medical College (Grant No. HG2020016-EC-1). 


\section{AUTHORS" CONTRIBUTIONS}

PD: Conceptualization, Data Curation, Formal Analysis, Writing - Original Draft, Writing - Review \& Editing. MG: Conceptualization, Writing - Original Draft, Writing Review \& Editing. YZ: Conceptualization, Writing - Review
\& Editing. XL: Data Curation, Formal Analysis, Writing Review \& Editing. XR: Data Curation, Formal Analysis, Writing - Review \& Editing. HW: Data Curation, Formal Analysis, Writing - Review \& Editing. JH: Data Curation, Formal Analysis, Writing - Review \& Editing.

\section{REFERENCES}

1 Medinger $M$, Drexler B, Lengerke $C$, Passweg J. Pathogenesis of acquired aplastic anemia and the role of the bone marrow microenvironment. Front Oncol. 2018;8:587. https://doi. org/10.3389/fonc. 2018.00587

2 Killick SB, Bown N, Cavenagh J, Dokal I, Foukaneli T, Hill A, et al. Guidelines for the diagnosis and management of adult aplastic anaemia. Br J Haematol. 2016;172(2):187-207. https:// doi.org/10.1111/bjh.13853

3 Bacigalupo A, Oneto R, Schrezenmeier H, Hochsmann B, Dufour C, Kojima S, et al. First line treatment of aplastic anemia with thymoglobuline in Europe and Asia: outcome of 955 patients treated 2001-2012. Am J Hematol. 2018;93(5):643-8. https:// doi.org/10.1002/ajh.25081

4 Scheinberg P, Wu CO, Nunez O, Young NS. Predicting response to immunosuppressive therapy and survival in severe aplastic anaemia. Br J Haematol. 2009;144(2):206-16. https://doi. org/10.1111/j.1365-2141.2008.07450.x

5 Shallis RM, Ahmad R, Zeidan AM. Aplastic anemia: etiology, molecular pathogenesis, and emerging concepts. Eur J Haematol. 2018;101(6):711-20. https://doi.org/10.1111/ejh.13153

6 Yang $W$, Wang $X$, Zhang $W$, Ying $H, X u$ Y, Zhang J, et al. Neutrophil-lymphocyte ratio and platelet-lymphocyte ratio are 2 new inflammatory markers associated with pulmonary involvement and disease activity in patients with dermatomyositis. Clin Chim Acta. 2017;465:11-6. https://doi.org/10.1016/j.cca.2016.12.007

7 Yolbas S, Yildirim A, Gozel N, Uz B, Koca SS. Hematological indices may be useful in the diagnosis of systemic lupus erythematosus and in determining disease activity in Behçet's disease. Med Princ Pract. 2016;25(6):510-6. https://doi.org/10.1159/000447948

8 Zhao Y, Shi Y, Shen H, Zhou D, Li L, Zhu J, et al. The prognostic value of platelet-lymphocyte ratio and neutrophil-lymphocyte ratio in the treatment response and survival of patients with peripheral T-cell lymphoma. Leuk Lymphoma. 2020;61(3):62330. https://doi.org/10.1080/10428194.2019.1700244

9 Wang S, Ma Y, Sun L, Shi Y, Jiang S, Yu K, et al. Prognostic significance of pretreatment neutrophil/lymphocyte ratio and platelet/lymphocyte ratio in patients with diffuse large b-cell lymphoma. Biomed Res Int. 2018;2018:9651254. https://doi. org/10.1155/2018/9651254

10 Camitta BM, Rappeport JM, Parkman R, Nathan DG. Selection of patients for bone marrow transplantation in severe aplastic anemia. Blood. 1975;45(3):355-63. PMID: 1090310

11 Li X, Shi J, Ge M, Shao Y, Huang J, Huang Z, et al. Outcomes of optimized over standard protocol of rabbit antithymocyte globulin for severe aplastic anemia: a single-center experience. PLoS One. 2013;8(3):e56648. https://doi.org/10.1371/journal.pone.0056648
12 Marsh JC, Bacigalupo A, Schrezenmeier H, Tichelli A, Risitano AM, Passweg JR, et al. Prospective study of rabbit antithymocyte globulin and cyclosporine for aplastic anemia from the EBMT Severe Aplastic Anaemia Working Party. Blood. 2012;119(23):5391-6. https://doi.org/10.1182/blood-2012-02-407684

13 Scheinberg P, Nunez O, Weinstein B, Scheinberg P, Biancotto $\mathrm{A}, \mathrm{Wu} \mathrm{CO}$, et al. Horse versus rabbit antithymocyte globulin in acquired aplastic anemia. N Engl J Med. 2011;365(5):430-8. https://doi.org/10.1056/NEJMoa1103975

14 Shah S, Jain P, Shah K, Patel K, Parikh S, Patel A, et al. Immunosuppressive therapy for aplastic anemia: a single-center experience from western India. Ann Hematol. 2019;98(1):41-6. https://doi.org/10.1007/s00277-018-3487-2

15 Scheinberg P, Cooper JN, Sloand EM, Wu CO, Calado RT, Young NS. Association of telomere length of peripheral blood leukocytes with hematopoietic relapse, malignant transformation, and survival in severe aplastic anemia. JAMA. 2010;304(12):135864. https://doi.org/10.1001/jama.2010.1376

16 Gu C, Zhu X, Qiao X, Zhai X, Shi W, Xie X. Multivariate logistic analysis of predictors of response to immunosuppressive therapy in children with aplastic anemia: a double-center study. Hematology. 2019;24(1):282-9. https://doi.org/10.10 80/16078454.2019.1565149

17 Sugimori $\mathrm{C}$, Chuhjo T, Feng X, Yamazaki H, Takami A, Teramura $M$, et al. Minor population of CD55-CD59- blood cells predicts response to immunosuppressive therapy and prognosis in patients with aplastic anemia. Blood. 2006;107(4):1308-14 https://doi.org/10.1182/blood-2005-06-2485

18 Jain R, Trehan A, Bansal D, Varma N. Aplastic anemia in children: how good is immunosuppressive therapy? Pediatr Hematol Oncol. 2019;36(4):211-21. https://doi.org/10.1080 /08880018.2019.1621970

19 Xie X, Zhao H, Qin D, Qiao X. Pharmacokinetics and pharmacodynamics of two antithymocyte globulins in treatment of pediatric aplastic anemia. Int J Clin Exp Med. 2015;8(3):434955. PMID: 26064352

20 Giannakoulas NC, Karakantza M, Theodorou GL, Pagoni M, Galanopoulos A, Kakagianni T, et al. Clinical relevance of balance between type 1 and type 2 immune responses of lymphocyte subpopulations in aplastic anaemia patients. $\mathrm{Br} J$ Haematol. 2004;124(1):97-105. https://doi.org/10.1046/j.13652141.2003.04729.x

21 S Shao YQ, Li XX, Ge ML, Shi J, Zhang J, Huang JB, et al. A longterm follow up study on 345 severe aplastic anemia patients treated with antithymocyte globulin/lymphoglobulin. Zhonghua Xue Ye Xue Za Zhi. 2013;34(1):30-5. Chinese. PMID: 23597461 